\title{
Substrate Selectivity of Exportin 5 and Dicer in the Biogenesis of MicroRNAs
}

\author{
E. LUND AND J.E. DAHLBERG \\ Biomolecular Chemistry, University of Wisconsin-Madison, Madison, Wisconsin 53706
}

\begin{abstract}
Each step in the biogenesis of microRNAs (miRNAs) depends on recognition of a correct substrate and efficient transport or processing of that RNA. Exportin5 (Exp5) and Dicer are proteins that mediate two key steps in this cascade, the nuclear export and cytoplasmic processing of microRNA precursor (pre-miRNAs). Xenopus laevis oocytes, eggs, and embryos constitute convenient experimental systems in which to study the substrate specificity of these proteins because specific RNAs or proteins can be injected directly into different subcellular compartments. We have used the Xenopus system and in vitro processing to define and compare the specificities of Exp5 and Dicer. Although both proteins act on many of the same substrates, we show that they recognize different structure elements of these RNAs. Our studies also revealed several unexpected activities. For example, Exp5 can mediate export of unspliced pre-mRNAs and excised lariat introns if these RNAs contain an aptamer sequence that itself is an Exp5 export substrate. Finally, we demonstrate that maturation of Xenopus oocytes into eggs leads to a large increase in Dicer activity, suggesting that miRNA biogenesis is subject to developmental control.
\end{abstract}

microRNAs (miRNAs) function in posttranscriptional control of gene expression through RISC (RNA-induced silencing complex)-mediated inhibition of translation, often accompanied by destabilization of the targeted mRNAs (for review, see Filipowicz et al. 2005; Valencia-Sanchez et al. 2006). miRNA biogenesis involves several coordinated processing and intracellular transport steps, some of which appear to be controlled during development and differentiation or in response to environmental stimuli (for review, see Bartel 2004; Du and Zamore 2005). These steps include (1) synthesis of primary transcripts containing one or more miRNAs, (2) processing of these transcripts by the RNase-III-like endonuclease Drosha (and associated proteins) to produce approximately 65- to 75-nucleotide-long incompletely base-paired hairpin RNAs (pre-miRNAs), (3) export of pre-miRNAs from the nucleus to the cytoplasm by the export factor exportin-5 (Exp5), (4) cleavage of the pre-miRNAs in the cytoplasm by a second doublestrand-specific RNase-III-like processing enzyme, Dicer, into duplexes about 22 nucleotides long, and (5) selection of one strand of the product duplex as the miRNA and incorporation into a ribonucleoprotein complex, the RISC (for review, see Murchison and Hannon 2004; Kim 2005). Here we describe our studies on the activities, substrate specificities, and developmental control of two of the key proteins that participate in this process, Exp5 and Dicer (steps 3 and 4).

We have asked what features of pre-miRNA substrates contribute to their export by Exp5 and their processing by Dicer. These hairpin RNAs have imperfectly base-paired stem regions about 22 nucleotides long, flanked by free $5^{\prime}$ and $3^{\prime}$ ends and an unpaired loop region. To test the importance of these structural elements in export and processing, we prepared ${ }^{32} \mathrm{P}$-labeled RNA substrates (by in vitro transcription; Grimm et al. 1997) in which these elements were either altered or deleted. We then monitored the fates of the variant RNAs in vivo, using microinjected Xenopus oocytes and embryos, or in vitro, using human recombinant Dicer.

\section{EXPORTIN 5}

We and other investigators previously demonstrated that Exp5 is responsible for export of pre-miRNAs from the nucleus to the cytoplasm of both $X$. laevis oocytes and mammalian cultured cells (Yi et al. 2003; Bohnsack et al. 2004; Lund et al. 2004), and that Exp5 binds directly to its RNA cargo in a RanGTP-dependent manner. Preferred export substrates resemble Drosha processing products (Lund et al. 2004; Zeng and Cullen 2004). Pre-miRNAs and other RNAs that are recognized by this export receptor (e.g., tRNA) all have a high degree of secondary structure, including short helices, indicating that double-stranded RNA structure is important to the interaction (Bohnsack et al. 2002; Calado et al. 2002; Gwizdek et al. 2003). Here, we asked what other features of the RNA contribute to its ability to serve as an export substrate for Exp5 in Xenopus oocytes.

\section{Pre-miRNA-related Export Substrates}

To determine whether Exp5 interacts with the free ends of the RNA cargos, we generated a pre-miRNA-like molecule that lacked free ends. This was done by ligating the ends of Drosophila melanogaster pre-let-7 RNA to each other, making a circular RNA (Fig. 1, left; top), which presumably maintained its secondary structure (Carrara et al. 1995). Upon injection into Xenopus oocyte nuclei, the gel-purified circular RNA was exported as efficiently as the linear pre-miRNA (Fig. 1, right; top panel). Likewise, a short imperfect miRNA-miRNA* duplex, generated by treatment of a human pre-miR-31 with Dicer, was exported as rapidly as was pre-miR-31 

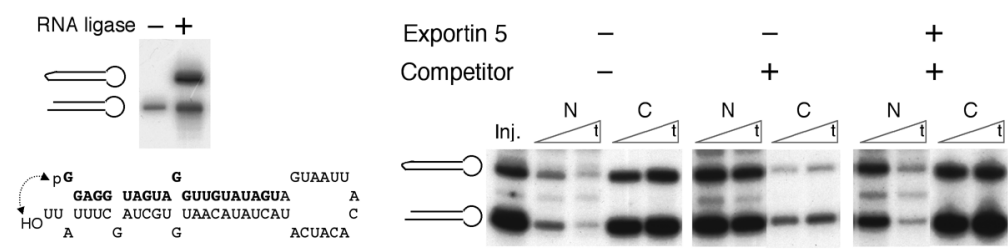

Dm pre-let-7
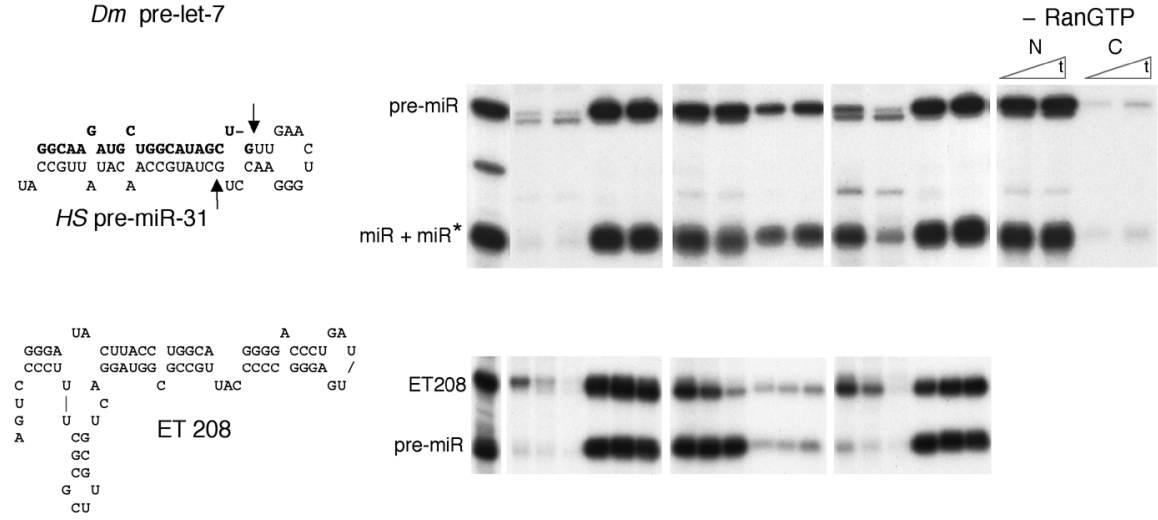

Figure 1. Substrate specificities of Xenopus Exp5. Structures on the left side show RNA substrates used in nuclear export experiments. The circular form of Dm pre-let-7 (top) was generated by treatment with RNA ligase (Carrara et al. 1995). Pre-miR-31 was digested with human recombinant Dicer, to produce the short miR-31/miR-31* duplex (indicated by arrows). ET-208 RNA is an aptamer selected for its ability to be exported in the presence of the M-protein of VSV (Grimm et al. 1997). Polyacrylamide gels display ${ }^{32} \mathrm{P}$ labeled RNAs that had been injected into Xenopus oocyte nuclei in the absence or presence of 0.5 pmole of unlabeled pre-miR-31 (top and middle panels) or ET-208 RNA (bottom panel) competitor RNAs and recombinant Exp5, or into oocytes lacking RanGTP $(*$ middle panel, right lanes) (Lund et al. 2004). Inj. denotes the injected RNAs, and N (nucleus) and C (cytoplasm) show the intracellular distributions of the RNAs with time $(t)$; the times were 25 and $40 \mathrm{~min}, 30$ and $60 \mathrm{~min}$, and 30, 60, and $120 \mathrm{~min}$ for the top, middle, and bottom panels, respectively.

(Fig. 1, right; middle panel). Export of both the circular RNA and the miRNA-miRNA* duplex was mediated by Exp5, as shown by the ability of an excess of unlabeled pre-miR-31 to inhibit it, and the ability of exogenous human recombinant Exp5 to reverse this inhibition (Fig. 1 , right; top and middle panels).

We confirmed that the appearance of the miRNA-miRNA* duplex RNA in the cytoplasm was due to active transport by depleting oocytes of RanGTP, an essential cofactor in Exp5-mediated nuclear export. This was accomplished by nuclear injection of recombinant RanGAP (to activate the GTPase function of Ran) and RanT24N (to inhibit RanGEF, the GTP-GDP exchange factor) (Izaurralde et al. 1997). In the absence of RanGTP, more than $95 \%$ of the short RNA duplex, as well as pre-miR-31, remained in the nucleus for at least 60 minutes (Fig.1, far right of middle panel), demonstrating that, like premiRNAs, the short duplex RNA is exported in a RanGTPdependent active process. Thus, neither the free ends nor the terminal loop region of a pre-miRNA is required for export by Exp5. This finding explains how Exp5 keeps duplex siRNAs out of the nucleus (Ohrt et al. 2006).

\section{Other Structured Exp5 Substrates}

Structured RNAs that are unrelated to pre-miRNAs can also be recognized and exported by Exp5. A novel example is ET-208 RNA (Fig. 1, left; bottom), an artificial, highly structured RNA aptamer that we selected from a library of sequences because of its ability to be exported from oocyte nuclei in the presence of the matrix protein of vesicular stomatitis virus (M protein) (Grimm et al. 1997). Although $\mathrm{M}$ protein potently inhibits export of both snRNAs and mRNAs (Petersen et al. 2000), it does not affect export of pre-miR-31 (not shown), suggesting that its export receptor, Exp5, could also mediate export of ET-208 RNA. Indeed, when co-injected into the same oocyte nuclei, ET208 RNA and pre-miR-31 strongly competed with each other for export, and this competition was obviated by injection of exogenous Exp5 (Fig. 1, bottom panel and data not shown). Moreover, we found that Exp5 binds directly to ET-208 RNA in a RanGTP-dependent manner (not shown).

Similarly, other workers have shown that the highly structured small RNAs, adenovirus VA1 RNA and human Y1 RNA (hY1) (Gwizdek et al. 2003), are export substrates for Exp5, further substantiating the versatility of this RNA-binding export receptor. However, we have found that some Exp5-mediated export (e.g., hY1 RNA) is subject to inhibition by M protein (Grimm et al. 1997; Rutjes et al. 2001; data not shown). This differential sensitivity of Exp5-mediated export to M protein may reflect the fact that ET-208 RNA was selected solely for its ability to be exported in the presence of the inhibitor, whereas hY1 RNA is complexed with specific RNA-binding protein(s) that might act as nuclear retention factors.

Both ET-208 RNA and pre-miR-31 are exported even when they lack free $5^{\prime}$ or $3^{\prime}$ ends (Fig. 1) (Grimm et al. 1997), suggesting that Exp5 recognizes internal structure(s) of the RNAs. Therefore, we asked whether these RNAs could serve as cis-acting elements to promote nuclear export of other RNA sequences. For this, we inserted ET-208 RNA or pre-miR-31 sequences into the intron of an AdML pre- 
mRNA model substrate. After injection of the chimeric premRNAs into oocyte nuclei, we monitored the intracellular distribution of the unspliced pre-mRNAs and excised intron lariats. As with other splicing/export systems, and as previously shown in oocytes (Pasquinelli et al. 1997), the AdML pre-mRNA and the excised intron lariat that lacked inserts were retained in the nucleus while spliced mRNA was exported (Fig. 2, left panel). However, both the chimeric pre-mRNA and the excised intron lariat were exported if they contained ET-208 RNA sequences (center panel); this export was dependent on Exp5, as it was inhibited by excess unlabeled competitor pre-miRNA and restored by exogenous Exp5 (data not shown). In contrast, the inserted premiR-31 sequence was unable to promote export of either the pre-mRNA or the excised intron lariat (Fig. 2, right panel). Thus, unlike ET-208 RNA, the structure of pre-miR-31 apparently is not recognized when embedded within a larger transcript, even though free ends are not needed for its export.

Thus, Exp5 is a versatile export receptor that recognizes RNA structure rather than the sequences or free ends of the substrate. The ability of Exp5 to recognize and export chimeric RNAs containing the ET-208 RNA sequence raises the possibility that this exportin-adapter pair could be used for nuclear export of other molecules.

\section{DICER}

Upon being exported to the cytoplasm by Exp5, premiRNAs are processed by Dicer to produce an imperfect RNA duplex (one strand of which will become the miRNA) with a 2-nucleotide single-stranded $3^{\prime}$ extension on each of its approximately 21- to 22-nucleotide-long strands (Bernstein et al. 2001; Lee et al. 2002; Zhang et al. $2002,2004)$. We investigated features of potential premiRNA substrates that might affect their ability to be processed by Dicer both in vitro, using human recombinant

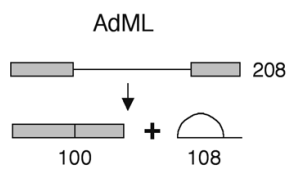

ET208-AdML or pre-mir31-AdML

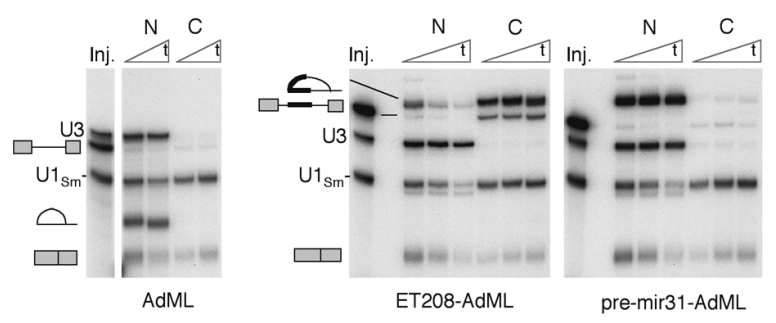

Figure 2. Exp5-mediated export of unspliced AdML pre-mRNA and its excised intron. Oocytes were injected in the nucleus with mixtures of control U3 and $\mathrm{U}_{\mathrm{Sm}^{-}}$RNAs and ${ }^{32} \mathrm{P}$-labeled preAdML RNA (Pasquinelli et al. 1997) with either no insert (left panels) or with ET-208 RNA or pre-miR-31 sequences (black bar) within the intron (middle and right panels, respectively). After 105 or 180 min incubation (left panel) or 105, 180, and 360 min incubation (middle and right panels), the nucleo-cytoplasmic distributions of precursors and splicing products were determined. Abbreviations are as in Fig. 1.
Dicer, and in vivo, using Xenopus oocytes and early embryos. The substrates, which resembled Drosophila pre-let-7 or human pre-miR-31, differed from each other in terms of the character of their ends, as well as the lengths of their duplex regions and terminal loops. In all of these experiments, the indicated cleavage sites were determined by direct nucleotide sequence analysis of the Dicer digestion products (not shown).

\section{Cleavage of Pre-miRNAs by Dicer In Vitro}

Free ends of Dicer substrates. We first asked whether free ends of the pre-miRNAs are needed for cleavage, by incubating the linear and circularized forms of pre-let-7 RNA (see Fig. 1) with human recombinant Dicer and analyzing the cleavage products by polyacrylamide gel electrophoresis. As expected, the control RNA with free ends was efficiently digested to yield fragments about 22 and 23 nucleotides long, arising from the stem, and a shorter fragment, about 14 nucleotides long, generated by cleavages near the end of the stem, at either side of the loop. In contrast, the circularized RNA was completely resistant to cleavage by Dicer (Fig. 3, top left panel), showing that at least one end of the RNA stem-loop structure is required for cleavage by Dicer. An earlier report indicating that free ends were not needed for Dicer cleavage used a substrate whose ends were base-paired but not ligated together (Zhang et al. 2002).

To study the effects of ends on the cleavage, we made several variants of pre-let7 RNA and pre-miR-31 (Fig. 3, right). First, we compared the products generated by Dicer digestion of pre-miR-31 with those of similar RNAs but with extra unpaired nucleotides at either the $5^{\prime}$ or 3' end (Fig. 3, RNAs 8, 9, and 10). The substrate with an extended $5^{\prime}$ end yielded the wild-type array of products (Fig. 3, bottom), whereas the substrate with a similar extension at its $3^{\prime}$ end yielded a complex mixture of products, and digestion was less efficient. The observed major sites of cleavage (denoted by horizontal bars on structures shown in Fig. 3, right) indicate that Dicer interacts with the free $3^{\prime}$ end and cuts the duplex at a fixed distance ( $\sim 22$ nucleotides away) (see Zhang et al. 2004).

Effects of stem length and sequence on Dicer cleavage. If the sites of cleavage are determined solely with reference to the $3^{\prime}$ extension at the base of the stem, premiRNA substrates with different length duplexes should be processed with similar efficiencies and yield products of predictable size. To test whether that was the case, we analyzed a series of synthetic substrates based on the sequence of Drosophila pre-let-7 (Fig. 3, right top) that were identical in and around the loop region but differed from each other by insertion of extra base pairs in various positions of the stem. All substrates with longer stems (except RNA 2) were cleaved less efficiently and, in most cases, yielded a heterogeneous array of products (Fig. 3, top; right panel), showing that the length of the stem also influences cleavage by Dicer. Moreover, digestion of substrates that had the same length but differed in the location of base-pair insertions resulted in different patterns of products (e.g., compare lanes 5 and 6), indicating that the 

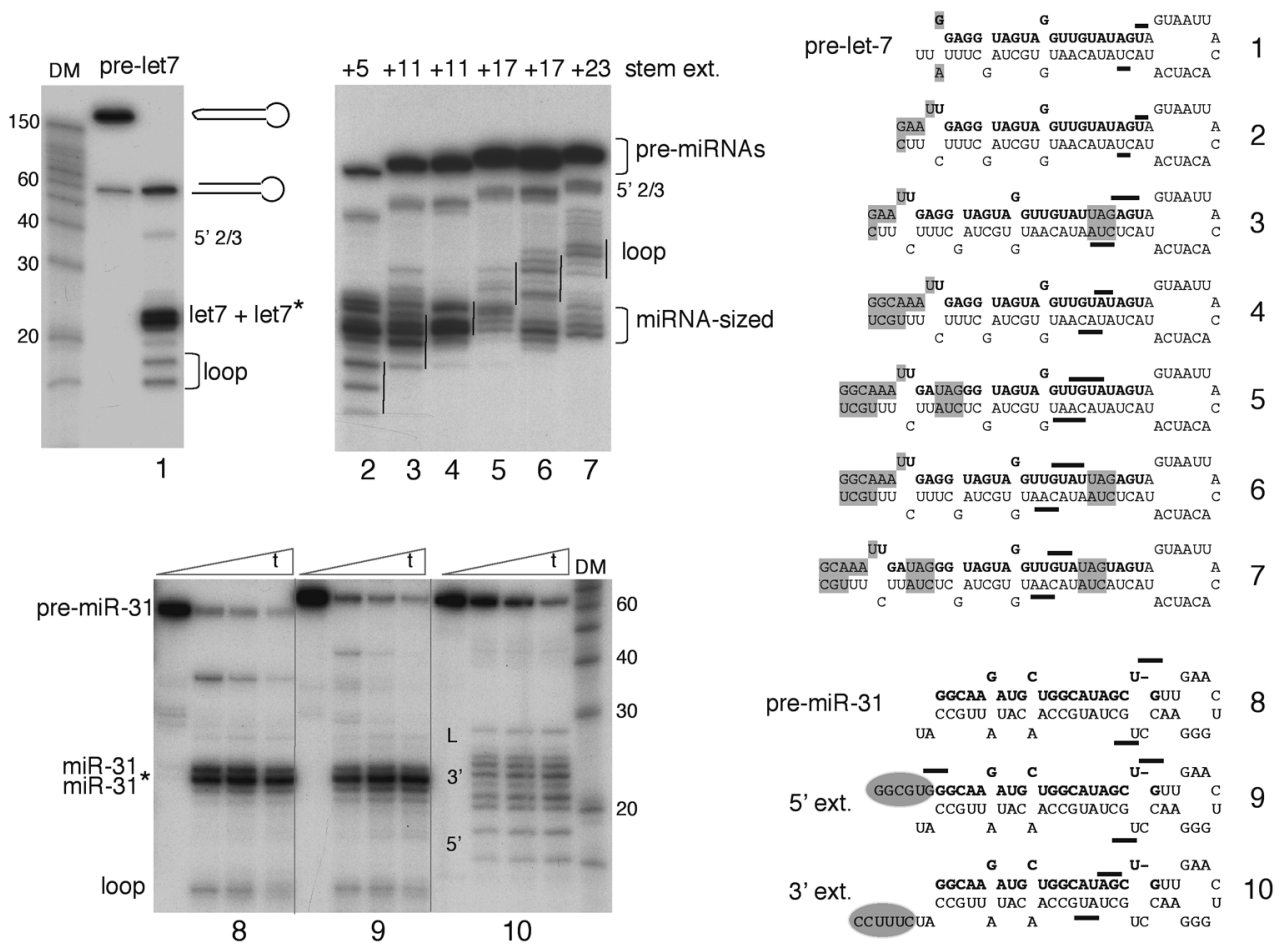

Figure 3. Role of the free ends of pre-miRNA and duplex lengths in cleavage by Dicer in vitro. Lanes 1-7 show products generated by incubation of $\sim 0.5$ pmole of ${ }^{32}$ P-labeled circularized or linear pre-let-7 RNA (Fig. 1), or variants of pre-let-7 (right side) with 1 unit Dicer (Gene Therapy Systems) for $60 \mathrm{~min}$ at $37^{\circ} \mathrm{C}$. Panels 8, 9, and 10 show the digestion products generated by incubation of premiR-31 and variant RNAs (right side) with Dicer for 0, 15, 30, and 60 min. Products were fractionated by electrophoresis in denaturing $20 \%$ polyacrylamide gels. Because the in vitro synthesized substrates were synthesized using either T7 or SP6 RNA polymerase, they all contain an extra non-encoded A at their $3^{\prime}$ ends (contributing or generating $3^{\prime}$ overhangs) which is not indicated in the figures.

sequence or structure within the stem region also could influence the site of cleavage.

Vertical lines in Figure 3 highlight the gel positions of fragments generated from the loop regions of the substrates. Apart from the short loop fragments, the shortest and most abundant cleavage products were miRNA-sized, in keeping with an initial cleavage at a fixed distance from the $3^{\prime}$ end. The products of all digests also included distinct, much larger fragments $\left(5^{\prime} 2 / 3\right.$ molecules $)$ that extended from the $5^{\prime}$ end of the stem through the loop to the cleavage site in the 3 ' side of the stem. The existence of these "deadend" partial digestion products indicates that Dicer cleaves first at the $3^{\prime}$ site, and that the duplex structure, which is needed for cleavage of the site in the $5^{\prime}$ side, has been disrupted in some of the substrate molecules.

Effects of loop size on Dicer cleavage. Although the site of cleavage clearly is determined in large part by measuring from the $3^{\prime}$ end of the stem, the effects of the length of the stem raise the possibility that interactions with the loop might also influence cleavage. Comparison of several variants of pre-let-7 showed that the size of the loop did not affect the site of cleavage (Fig. 4, top). However, loop size did affect the efficiency of cleavage, as shown by the reduced rate of digestion of the substrate with only 4 nucleotides (right panel). This reduction in efficiency may reflect distortion of the cleavage site near the end of the duplex, due to constraints imposed by the tight bend of the RNA backbone within the small loop.
As an RNase-III-like enzyme, Dicer cleaves RNAs in duplex regions, but it also fixes the site of cleavage at about 22 nucleotides from the $3^{\prime}$ end of the stem of the substrate (Fig. 3) (Zhang et al. 2004; Vermeulen et al. 2005). Therefore, duplexes shorter than 21 or 22 nucleotides would not be expected to be substrates for this enzyme. We tested this prediction by incubating Dicer with short hairpin RNAs (shRNAs) that had perfectly complementary stems 22, 21, or 19 bp long (RNAs 4, 5, and 6 at the bottom of Fig. 4). Surprisingly, the shRNA substrate with 22 bp (RNA 4) was cleaved much less efficiently than the corresponding pre-miRNA of similar length (RNA 3). This difference indicates that the unpaired nucleotides in the stems of pre-miRNAs may facilitate the positioning of the scissile bonds in appropriate RNase III active sites of the enzyme. As predicted, shortening the stem to 21 or 19 bp (RNAs 5 and 6) while maintaining the overall length of the shRNA (49 nucleotides) further reduced or completely abolished cleavage by Dicer.

shRNA substrates. Using similar substrates in their pioneering study on siRNA expression, Brummelkamp et al. (2002) reported that an shRNA with a stem of only 19 bp and a loop of 9 nucleotides (19/9) could be processed to make functional siRNAs, whereas substrates with shorter loops (19/7 or 19/5) could not. That led them to conclude that a 19-bp stem was sufficient for a substrate for Dicer, if the adjacent loop was 9 nucleotides long. 

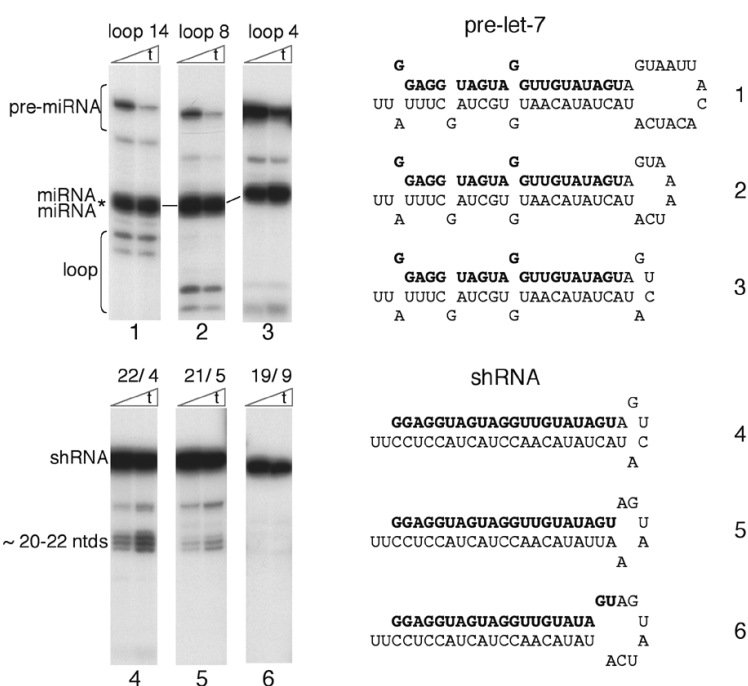

Figure 4. Influence of loop size and stem length of pre-miRNAs and shRNAs on cleavage by Dicer in vitro. ${ }^{32} \mathrm{P}$-labeled variants of pre-let-7 and shRNAs (right side) were incubated with Dicer for 5 or $10 \mathrm{~min}$ (top panels) or 10 and $30 \mathrm{~min}$ (bottom panels) as in Fig. 3. Numbers above the bottom panels indicate the number of base pairs in the stem and the number of nucleotides in the loop of the shRNAs, respectively.

However, we note that two additional base pairs could be formed by nucleotides at the ends of the 9-nucleotide loop that they used, which would extend the stem to 21 pairs (and reduce the loop to 5). In the 19/9 substrate used here, extension of the stem was not possible and cleavage did not occur, showing that more than $19 \mathrm{bp}$ are required for cleavage by Dicer. We conclude that the minimum stem length of an shRNA (and very likely a pre-miRNA) is 21 or 22 nucleotides.

Taken together, our data on the substrate requirements for cleavage of hairpin RNAs indicate that Dicer fixes the site of cleavage in a duplex region by measuring from the free $3^{\prime}$ end at the base of a stem, and that binding of the enzyme to the substrate is increased by interaction with the loop and perhaps the backbone of the stem. This interpretation is consistent with recent in vivo and in vitro studies of other workers (Zhang et al. 2004; Vermeulen et al. 2005; Chang et al. 2006), and it fits the model for substrate recognition based on the crystal structure of Dicer from Giardia intestinalis (MacRae et al. 2006). In that model, binding of the short $3^{\prime}$ overhang in the PAZ domain pocket at one end of the enzyme positions the duplex near the RNase III domains, allowing cleavage about 22 bp away. A mechanism for recognition of the loop by the minimal enzyme used in that study is unclear.

Non-pre-miRNA substrates. Finally, we asked whether other structured RNAs that are exported by Exp5 could also bind to, and be cleaved by, Dicer. As an indicator of binding, we measured the ability of an RNA to compete for cleavage of pre-let-7 RNA. hY1 RNA, which has a very short terminal stem (see Rutjes et al. 2001), had no effect on cleavage of pre-miR-31 (not shown). However, VA1 RNA of adenovirus 2, which has a very long, inter- rupted stem (Fig. 5, left), was an effective inhibitor, as has recently been reported ( $\mathrm{Lu}$ and Cullen 2004; Andersson et al. 2005). Likewise, ET-208 RNA competed for cleavage of pre-miRNA, albeit less efficiently (Fig. 5, top panels). However, neither VA1 RNA nor ET-208 RNA was a good substrate for cleavage; we detected only a very low level of VA1 cleavage, in agreement with other studies (Andersson et al. 2005; Sano et al. 2006), but we never observed any cleavage of ET-208 RNA (Fig. 5, lower panels). We propose that these RNAs bind via their $3^{\prime}$ overhangs to the PAZ domain and via their stem and loops to other structures of human Dicer including the dsRBD (Zhang et al. 2004; MacRae et al. 2006). Their ability to act as competitors without being cleaved indicates that they can do so without placing scissile bonds near the RNase III processing center of the enzyme. In vivo, none of these structured RNAs appeared to interfere with Dicer activity in Xenopus oocytes or eggs (not shown), perhaps because of interactions with other, more abundant or more avidly binding proteins. This is unlike the suppression of Dicer activity observed in VA1 RNA-producing mammalian cells (Lu and Cullen 2004; Andersson et al. 2005).

\section{Processing by Dicer In Vivo}

To study Dicer activity in vivo, we injected several synthetic pre-miRNAs and stem-loop RNAs into either the nucleus or cytoplasm of Xenopus oocytes or early embryos. As shown above (Fig. 1) and reported previously (Lund et al. 2004), pre-miRNAs injected into nuclei are rapidly exported into the cytoplasm where they appear to remain stable. However, these pre-miRNAs actually were processed in the cytoplasm, albeit slowly (Fig. 6, left

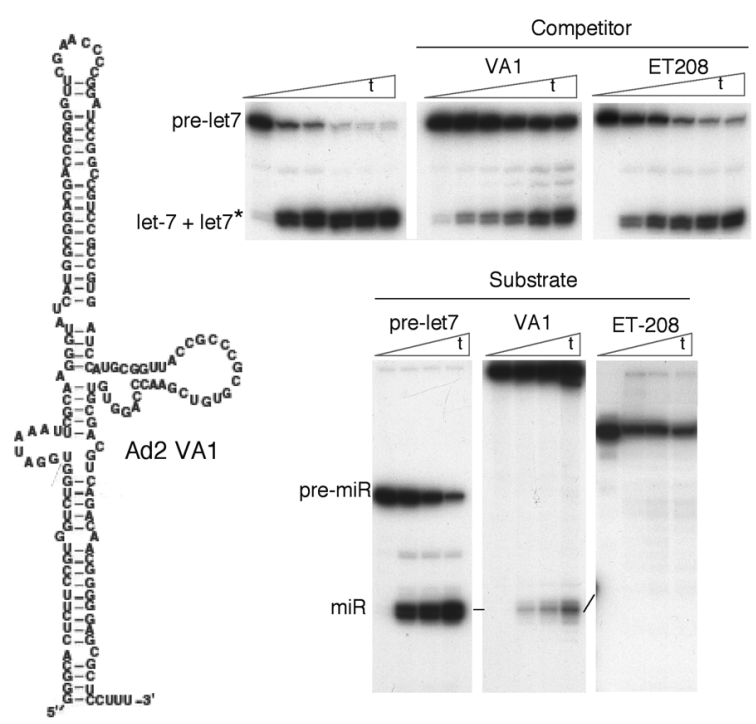

Figure 5. Interactions of structured RNAs with Dicer in vitro. The structure of VA1 RNA was adapted from Sano et al. (2006). The top panels show the products of Dicer cleavage of ${ }^{32} \mathrm{P}$ labeled pre-let-7 upon incubation for $0,5,15,30,60$, and $90 \mathrm{~min}$ in the absence or presence of 20 pmoles ( 40-fold molar excess) of the indicated competitor RNAs. The bottom panels show the products of Dicer digestion of ${ }^{32} \mathrm{P}-$ labeled pre-let7, VA1, or ET208 RNAs for 0, 5, 15, and 30 min, as in Fig. 3. 
panel), to yield miRNA-miRNA* duplexes identical to the cleavage products generated in vitro. After 2 hours, only a small percentage of the injected pre-miRNA was cleaved, and even after overnight incubation, no more than $10-15 \%$ of the pre-miRNA substrates had been processed to miRNA. Thus, stage VI oocytes have detectable but low levels of Dicer activity.

To rule out that Dicer digestion products might have been produced in the nucleus and then rapidly exported to the cytoplasm by Exp5, we blocked nuclear export by depleting the oocytes of RanGTP (cf. Fig. 1). Under these conditions, the injected pre-miR-31 remained largely in the nucleus, but no processed miRNA was detected there (Fig. 6, second panel); thus, nuclei of stage VI oocytes are devoid of detectable amounts of Dicer activity, in agreement with the intracellular localization of Dicer in mammalian cells (Billy et al. 2001). Furthermore, the same extent of processing was observed regardless of whether the pre-miRNA substrate was injected into the nucleus or the cytoplasm (third panel).

\section{Changes in Dicer Activity during Early Development}

Incubation of stage VI oocytes with progesterone triggers their maturation into eggs. Upon breakdown of the germinal vesicle (nucleus), many hitherto dormant activities can be observed (Mendez and Richter 2001). When oocytes were matured, we observed a large increase in Dicer activity (Fig. 6, far right panel), with more pre-miR31 RNA processed in matured oocytes within 30 minutes than during 4 hours in stage VI oocytes. Quantification of processing in several batches of oocytes showed a consistent five- to eightfold increase in Dicer activity upon maturation. Both miRNA and miRNA* products were detected, indicating that selection of one strand into a mature RISC might be inefficient. Comparable results were observed upon microinjection of several different pre-miRNAs (not shown).

It is unclear whether the large increase in Dicer activity that occurs upon breakdown of the germinal vesicle results from activation of preexisting enzyme or removal of an inhibitor, or from de novo synthesis of either Dicer or a cofactor(s) such as Xlrbpa (Eckmann and Jantsch
1997), the likely Xenopus homolog of TRBP (Haase et al. 2005; Maniataki and Mourelatos 2005) or PACT (Lee et al. 2006). A similar increase in Dicer activity was observed in a comparison of whole-cell extracts of stage VI oocytes, matured oocytes, and embryos (not shown), indicating that activation was not due simply to release of a sequestered cofactor from the nucleus.

We then asked whether the endogenous Dicer present in matured oocytes had the same substrate specificity as the human enzyme that we used in vitro. To make this comparison, matured oocytes were injected with several of the variant substrates that had been tested in vitro (Figs. 3 and 4). In general, processing in vivo matched the cleavage observed in vitro, but increased stringency in vivo was observed in several cases (Fig. 7). For example, the variant with only a 4-nucleotide-long loop that was cleaved well in vitro (RNA 3 in Fig. 4) was a poor substrate in vivo (middle panel, right lanes). Likewise, the RNA with a 14-nucleotide loop and 23-nucleotide stem extension (RNA 7 in Fig. 3) was not processed at all in vivo (Fig. 7, middle panel; left lanes). The shRNAs with 22 and 21 base-paired stems (RNAs 4 and 5 of Fig. 4) appeared to be better substrates in vivo than in vitro, but the one with only 19 bp (RNA 6) was not (Fig. 7, right panel). Comparable results were obtained in stage VI oocytes, but the extent of processing (and thus the signal) was considerably lower (data not shown). Changes in stringency of substrate recognition, while not great, could significantly influence the fidelity of processing events in vivo. The changes presumably result from interactions with one or more cofactors that facilitate Dicer activity.

The great increase in Dicer activity upon germinal vesicle breakdown (Fig. 6) presumably could support production of large quantities of miRNAs immediately after fertilization, during the early cleavage stage of Xenopus embryogenesis. However, we and other workers have observed that biogenesis of novel miRNAs is coincident with the mid-blastula transition (MBT), when zygotic transcription is activated (Newport and Kirshner 1982). At that time ( $\sim 7$ hours after fertilization), abundant accumulation of miR-427 is observed (Watanabe et al. 2005; E. Lund et al., in prep.).

We asked whether the early cleavage embryos, despite their very short cell cycle ( $\sim 25-30$ minutes), were still able

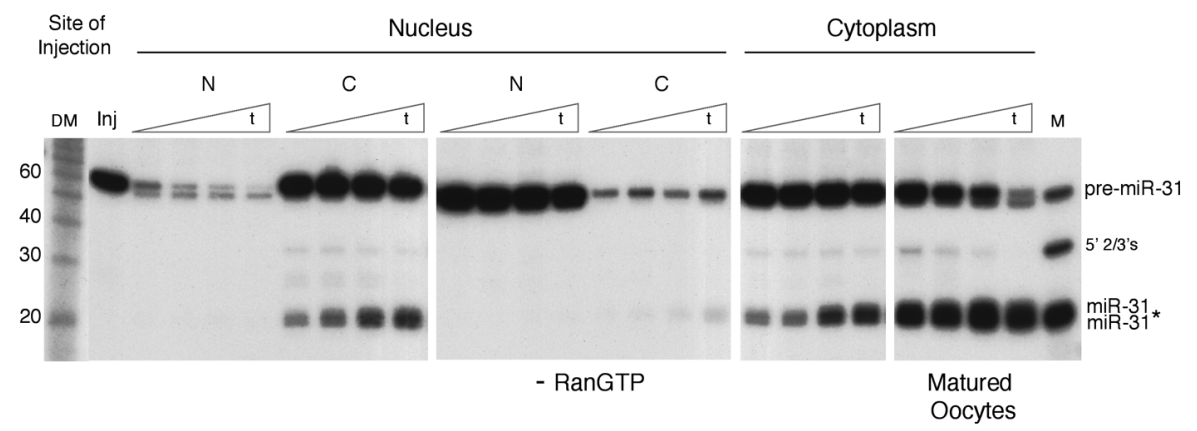

Figure 6. Processing of pre-miRNA in stage VI and matured Xenopus oocytes. ${ }^{32} \mathrm{P}$-labeled pre-miR-31 was injected into the nuclei (left two panels) or cytoplasms (third panel) of stage VI oocytes without or with prior depletion of RanGTP (-RanGTP) (Lund et al. 2004). After $0.5,1,2$, or 4 hours incubation at $23^{\circ} \mathrm{C}$, the nucleo-cytoplasmic distributions of RNA products were determined as in Fig. 3 . The far right panel shows the products resulting from injection into oocytes that had been matured by prior incubation (16 hours) with $10 \mu \mathrm{g} / \mathrm{ml}$ progesterone. 


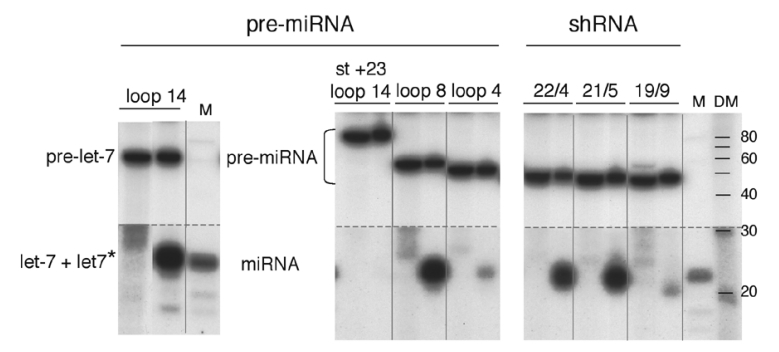

Figure 7. Influence of loop size and stem length on cleavage by Dicer in vivo. ${ }^{32} \mathrm{P}$-labeled pre-miRNA and shRNA substrates (structures shown in Figs. 3 and 4) were injected into matured oocytes and analyzed 0 and 4 hours later for evidence of cleavage by endogenous Xenopus Dicer. Because several of the RNAs tested were poor substrates (despite the increased Dicer activity observed upon oocyte maturation), detection of the products was enhanced by $\sim 10$ times longer exposure of the bottom portions of the gels (below the dashed line).

to fully process pre-miRNAs and assemble them into RISCs. For this, we injected in vitro synthesized pre-miR427 RNA into one- or two-cell embryos and observed its processing during normal development (Fig. 8). Mature miR-427 was visible already at the 32-cell stage $(\sim 1.5-2$ hours after injection, or 3.5 hours post fertilization) and continued to accumulate through MBT and gastrulation (12 hours). The rate of disappearance of the injected pre-miR427 appeared to accelerate in the approximately 2 hours prior to MBT, but that was not matched by a concomitant increase in the levels of mature miR-427, suggesting that processing by Dicer may have been saturated (although increased flux of material through mature miRNA cannot

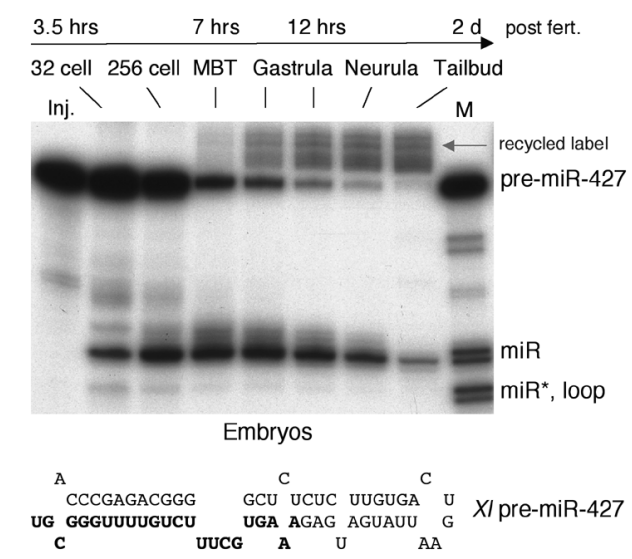

Figure 8. Processing of pre-miR-427 during early Xenopus embryogenesis. Between 1.5 and 2 hours after fertilization of Xenopus eggs (Newport and Kirshner 1982), ${ }^{32}$ P-labeled premiR-427 (structure at the bottom) was injected into one- or twocell embryos. Embryonic development was monitored as indicated, and at the indicated stages of embryonic development, RNAs were isolated from pools of four embryos and one embryo-equivalents of total RNAs were analyzed in a 20\% denaturing polyacrylamide gel. After the mid-blastula transition (MBT), some of the labeled nucleotides from the injected RNA were recycled into newly synthesized tRNA molecules. The time line at the top indicates times after fertilization. The gel mobilities of Dicer digestion products (miR-427 and miR-427* and the loop) are indicated on the right. be excluded). It is likely that the amount of accumulated miRNA represents a steady state that was achieved by simultaneous production and degradation of both the injected ( ${ }^{32} \mathrm{P}$-labeled) and endogenous (unlabeled) miR427. We note that only miR-427, but not its complementary strand, miR-427* (which migrates faster in the gel), accumulated, strongly indicating that the Dicer digestion products had been incorporated into mature RISCs. Importantly, this suggests that miRNA-mediated repression of translation may be operable during the earliest stages of embryonic development (E. Lund et al., in prep.).

\section{CONCLUSIONS}

Exp5 and Dicer are two proteins that are essential for the generation of microRNAs. The results discussed here demonstrate that, although both factors are able to accommodate a good deal of variability in their cargos or substrates, they also exhibit some strict substrate preferences or requirements. Undoubtedly, this level of stringency functions to reduce the level of spurious RNAs that could act like miRNAs, with detrimental consequences. Our data also demonstrate that the activity of at least one key enzyme, and probably several others (Thomson et al. 2006), involved in miRNA biogenesis is developmentally controlled. These findings highlight the importance of controlling the amounts of miRNAs that can be produced and function at various stem cell and embryonic stages, and probably in adult tissues, as well.

\section{ACKNOWLEDGMENTS}

This work was supported by National Institutes of Health grant GM 30220. We thank Susanne Imboden Blaser, Christian Bille Jendresen, Philipp Müller, Lei Tong, and David Trittle for help with RNA substrate preparation and in vitro Dicer assays.

\section{REFERENCES}

Andersson M.G., Haasnoot P.C., Xu N., Berenjian S., Berkhout B., and Akusjärvi G. 2005. Suppression of RNA interference by adenovirus virus-associated RNA. J. Virol. 79: 9556.

Bartel D.P. 2004. MicroRNAs: Genomics, biogenesis, mechanism, and function. Cell 116: 281.

Bernstein E., Caudy A.A., Hammond S.M., and Hannon G.J. 2001. Role for a bidentate ribonuclease in the initiation step of RNA interference. Nature 409: 363.

Billy E., Brondani V., Zhang H., Muller U., and Filipowicz W. 2001. Specific interference with gene expression induced by long, double-stranded RNA in mouse embryonal teratocarcinoma cell lines. Proc. Natl. Acad. Sci. 98: 14428.

Bohnsack M.T., Czaplinski K., and Görlich D. 2004. Exportin 5 is a RanGTP-dependent dsRNA-binding protein that mediates nuclear export of pre-miRNAs. RNA 10: 185.

Bohnsack M.T., Regener K., Schwappach B., Saffrich R., Paraskeva E., Hartmann E., and Görlich D. 2002. Exp5 exports eEF1A via tRNA from nuclei and synergizes with other transport pathways to confine translation to the cytoplasm. EMBO J. 21: 6205.

Brummelkamp T.R., Bernards R., and Agami R. 2002. A system for stable expression of short interfering RNAs in mammalian cells. Science 296: 550.

Calado A., Treichel N., Muller E.C., Otto A., and Kutay U. 2002. Exportin-5-mediated nuclear export of eukaryotic elongation factor 1A and tRNA. EMBO J. 21: 6216. 
Carrara G., Calandra P., Fruscoloni P., and Tocchini-Valentini G.P. 1995. Two helices plus a linker: A small model substrate for eukaryotic RNase P. Proc. Natl. Acad. Sci. 92: 2627.

Chang K., Elledge S.J., and Hannon G.J. 2006. Lessons from Nature: microRNA-based shRNA libraries. Nat. Methods. 3: 707.

Du T. and Zamore P.D. 2005. microPrimer: The biogenesis and function of microRNA. Development 132: 4645.

Eckmann C.R. and Jantsch M.F. 1997. Xlrbpa, a doublestranded RNA-binding protein associated with ribosomes and heterogeneous nuclear RNPs. J. Cell Biol. 138: 239.

Filipowicz W., Jaskiewicz L., Kolb F.A., and Pillai R.S. 2005. Post-transcriptional gene silencing by siRNAs and miRNAs. Curr. Opin. Struct. Biol. 15: 331.

Grimm C., Lund E., and Dahlberg J.E. 1997. Selection and nuclear immobilization of exportable RNAs. Proc. Natl. Acad. Sci. 94: 10122.

Gwizdek C., Ossareh-Nazari B., Brownawell A.M., Doglio A., Bertrand E., Macara I.G., and Dargemont C. 2003. Exportin5 mediates nuclear export of minihelix-containing RNAs. $J$. Biol. Chem. 278: 5505.

Haase A.D., Jaskiewicz L., Zhang H., Laine S., Sack R., Gatignol A., and Filipowicz W. 2005. TRBP, a regulator of cellular PKR and HIV-1 virus expression, interacts with Dicer and functions in RNA silencing. EMBO Rep. 6: 961.

Izaurralde E., Kutay U., von Kobbe C., Mattaj I.W., and Görlich D. 1997. The asymmetric distribution of the constituents of the Ran system is essential for transport into and out of the nucleus. $E M B O J$. 16: 6535.

Kim V.N. 2005. MicroRNA biogenesis: Coordinated cropping and dicing. Nat. Rev. Mol. Cell Biol. 6: 376

Lee Y., Jeon K., Lee J.T., Kim S., and Kim V.N. 2002. MicroRNA maturation: Stepwise processing and subcellular localization. $E M B O J .21: 4663$.

Lee Y., Hur I., Park S.Y, Kim Y.K., Suh M.R., and Kim V.N. 2006. The role of PACT in the RNA silencing pathway. EMBO J. 25: 522

Lu S. and Cullen B.R. 2004. Adenovirus VA1 noncoding RNA can inhibit small interfering RNA and MicroRNA biogenesis. J. Virol. 78: 12868.

Lund E., Güttinger S., Calado A, Dahlberg J.E., and Kutay U. 2004. Nuclear export of microRNA precursors. Science 303: 95.

MacRae I.J., Zhou K., Li F., Repic A., Brooks A.N., Cande W.Z., Adams P.D., and Doudna J.A. 2006. Structural basis for double-stranded RNA processing by Dicer. Science 311: 195.

Maniataki E. and Mourelatos Z. 2005. A human, ATP-independent, RISC assembly machine fueled by pre-miRNA. Genes Dev. 19: 2979.

Mendez R. and Richter J.D. 2001. Translational control by CPEB: A means to the end. Nat. Rev. Mol. Cell Biol. 2: 521.

Murchison E.P. and Hannon G.J. 004. miRNAs on the move:
miRNA biogenesis and the RNAi machinery. Curr. Opin. Cell. Biol. 16: 223.

Newport J. and Kirschner M. 1982. A major developmental transition in early Xenopus embryos. I. Characterization and timing of cellular changes at the midblastula stage. Cell 30: 675 .

Ohrt T., Merkle D., Birkenfeld K., Echeverri C.J., and Schwille P. 2006. In situ fluorescence analysis demonstrates active siRNA exclusion from the nucleus by Exportin 5. Nucleic Acids Res. 34: 1369.

Pasquinelli A.E., Ernst R.K., Lund E., Grimm C., Zapp M.L, Rekosh D., Hammarskjöld M.L., and Dahlberg J.E. 1997. The constitutive transport element (CTE) of Mason-Pfizer monkey virus (MPMV) accesses a cellular mRNA export pathway. EMBO J. 16: 7500.

Petersen J.M., Her L.S, Varvel V., Lund E., and Dahlberg J.E. 2000. The matrix protein of vesicular stomatitis virus inhibits nucleocytoplasmic transport when it is in the nucleus and associated with nuclear pore complexes. Mol. Cell. Biol. 20: 8590.

Rutjes S.A., Lund E., van der Heijden A., Grimm C., van Venrooij W.J., and Pruijn G.J. 2001. Identification of a novel cis-acting RNA element involved in nuclear export of hY RNAs. RNA 7: 741.

Sano M., Kato Y., and Taira K. 2006. Sequence-specific interference by small RNAs derived from adenovirus VAI RNA. FEBS Lett. 580: 1553.

Thomson J.M., Newman M., Parker J.S., Morin-Kensicki E.M., Wright T., and Hammond S.M. 2006. Extensive post-transcriptional regulation of microRNAs and its implications for cancer. Genes Dev. 20: 2202.

Valencia-Sanchez M.A., Liu J., Hannon G.J., and Parker R. 2006. Control of translation and mRNA degradation by miRNAs and siRNAs. Genes Dev. 20: 515.

Vermeulen A., Behlen L., Reynolds A., Wolfson A., Marshall W.S., Karpilow J., and Khvorova A. 2005. The contributions of dsRNA structure to Dicer specificity and efficiency. $R N A$ 11: 674 .

Watanabe T., Takeda A., Mise K., Okuno T., Suzuki T., Minami N., and Imai H. 2005. Stage-specific expression of microRNAs during Xenopus development. FEBS Lett. 579: 318.

Yi R., Qin Y., Macara I.G., and Cullen B.R. 2003. Exportin-5 mediates the nuclear export of pre-microRNAs and short hairpin RNAs. Genes Dev. 17: 3011.

Zeng Y. and Cullen B.R. 2004. Structural requirements for premicroRNA binding and nuclear export by Exportin 5. Nucleic Acids Res. 32: 4776.

Zhang H., Kolb F.A., Brondani V., Billy E., and Filipowicz W. 2002. Human Dicer preferentially cleaves dsRNAs at their termini without a requirement for ATP. EMBO J. 21: 5875 .

Zhang H., Kolb F.A., Jaskiewicz L., Westhof E., and Filipowicz W. 2004. Single processing center models for human Dicer and bacterial RNase III. Cell 118: 57. 


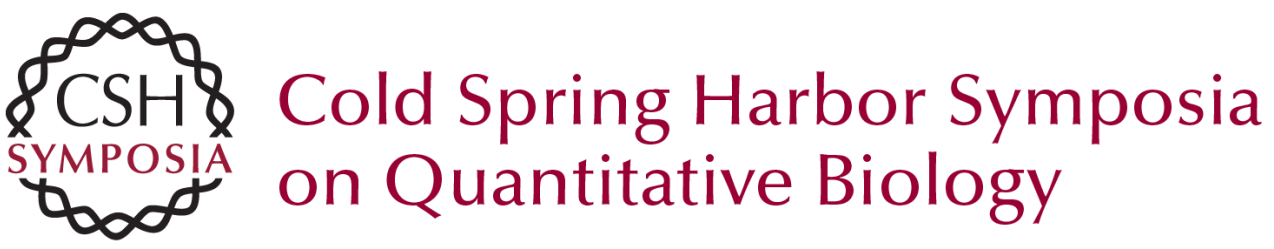

\section{Substrate Selectivity of Exportin 5 and Dicer in the Biogenesis of MicroRNAs}

E. LUND and J.E. DAHLBERG

Cold Spring Harb Symp Quant Biol 2006 71: 59-66

Access the most recent version at doi:10.1101/sqb.2006.71.050

References This article cites 39 articles, 27 of which can be accessed free at: http://symposium.cshlp.org/content/71/59.full.html\#ref-list-1

License

Email Alerting Receive free email alerts when new articles cite this article - sign up in Service the box at the top right corner of the article or click here. 